\title{
Magnetic particle imaging signal acquisition using second harmonic detection of magnetic nanoparticles
}

\author{
Muhammad Mahadi Abdul Jamil ${ }^{1}$, Abdulkadir Abubakar Sadiq ${ }^{2}$, Muhammad Shukri Ahmad ${ }^{3}$, \\ Noordin Asimi Mohd Noor ${ }^{4}$, Nur Adilah Abd Rahman ${ }^{5}$, Nurmiza Othman \\ ${ }^{1,2,5,6}$ Department of Electronics Engineering, Universiti Tun Hussein Onn Malaysia, Malaysia \\ ${ }^{3}$ Pusat Pengajian Diploma, Universiti Tun Hussein Onn Malaysia, Malaysia \\ ${ }^{4}$ Faculty of Innovative Design and Technology, Universiti Sultan Zainal Abidin, Malaysia
}

\begin{tabular}{l} 
Article Info \\
\hline Article history: \\
Received Dec 11, 2018 \\
Revised Feb 12, 2019 \\
Accepted Feb 27, 2019 \\
\hline
\end{tabular}

Keywords:

Magnetic nanoparticles Magnetic particle imaging Second harmonic detection Tomographic images

\begin{abstract}
This paper presents an approach for acquiring a magnetic particle imaging (MPI) signal, by utilizing the second harmonic detection of the magnetic nanoparticles tracers. An MPI signal with high signal-to-noise ratio (SNR) is crucial for high spatial resolution images that reveals the distribution of the tracers in a target area. Samples of Resovist and Perimag nanoparticles tracers were prepared in liquid and immobilised form, which were placed at some distances under the receiver coil of the single-sided MPI scanner. The samples were exposed to the excitation magnetic field generated at 22.8 $\mathrm{kHz}$ and a static gradient field generated with a direct current of $2 \mathrm{~A}$. The non-linear magnetization response of the tracers for each spatial position is recorded in the form of voltage signal by a gradiometer pickup coil, with the second harmonic signal being extracted by a resonance circuit. The results obtained revealed that a sufficient signal from the tracers is recorded at up to $25 \mathrm{~mm}$ under the pickup coil, with Perimag samples inducing higher signals as compared to Resovist. The dependence of the DC gradient field on the second harmonic signal shows that the peak signal amplitude for Resovist and Perimag particles as $\pm 5 \mathrm{mT}$ and $\pm 6 \mathrm{mT}$ respectively. Additionally, the second harmonic signal amplitude increases exponentially with an increase in the excitation magnetic field. Thus, the results obtained shows the potential of this approach in acquiring high SNR MPI signals at low excitation frequency, which could be vital in reconstructing the contour images of the tracers, particularly in sentinel lymph node biopsy (SLNB) for breast cancer diagnosis.
\end{abstract}

Copyright $\odot 2019$ Institute of Advanced Engineering and Science. All rights reserved.

\section{Corresponding Author: \\ Muhammad Mahadi Abdul Jamil, Department of Electronics Engineering, Universiti Tun Hussein Onn Malaysia, Johor, Malaysia. \\ Email:mahadi@uthm.edu.my}

\section{INTRODUCTION}

Magnetic particle imaging (MPI) is a new imaging method which exploits the non-linear magnetization response of magnetic nanoparticles (MNPs) tracers to reveal their distribution within a target [1]. The MNPs are excited by the AC magnetic modulation field generated by the MPI scanner and respond with a non-linear magnetization. The magnetization (usually called MPI signal), contains not only the fundamental excitation frequency, but also its harmonics occurring at multiples of the excitation frequencies [2-5]. Harmonic detection of MNPs is a non-linear AC susceptibility detection approach, where a sufficient excitation field with amplitude H0 is applied to magnetize the MNPs. The influence of the excitation field is then removed by a Bandstop filter, and the amplitude of the harmonics from the non-linear 
magnetization response of the particles is utilized for the MNPs image reconstruction. Before image reconstruction, the fast Fourier transform (FFT) of the signal is determined, to convert the signal from time domain to frequency domain, where the second harmonic signal is certainly picked by a resonant circuit and logged into the computer by a data acquisition device (DAQ) $[3,6]$.

In this paper, an alternative approach of acquiring the second harmonic MPI signal is proposed and demonstrated experimentally. Initially, a gradiometer coil sensor is employed as the MPI signal receiver in order to cancel out the interference of the excitation field and the earth's magnetic field. Then, a Bandstop filter is used to further attenuate the component of the excitation, so as to have a sufficient signal with high signal-to-noise ratio (SNR). Finally, the second harmonic signal amplitudes are extracted by the resonant circuit designed for second harmonic detection. These steps of MPI signal acquisition yields a high SNR signal which increases the spatial resolution of the reconstructed images, which could help in sentinel lymph node biopsy (SLNB) for breast cancer diagnosis.

\section{DETECTION METHODS OF MNPS}

Thus far, there are three techniques for MNPs detection as reported in [7], which are: AC susceptibility (ACS), magnetic relaxation (MRX) and remanence-based measurement methods. In ACS, a periodic excitation field $H=H_{0} \sin \left(2 \pi f_{0} t\right)$ having an excitation frequency $f_{0}$ and amplitude $H_{0}$, is applied to the MNPs. When the value of $H_{0}$ is small such that the Langevin parameter is less than unity, the magnetisation produced by the MNPs is within the linear region. This situation is called linear ACS, and the signal frequency cannot be differentiated from the excitation frequency. But when the $H_{0}$ is large, a weak signal is obtained, that needs to be extracted from the large $H_{0}$. The extraction process utilizes the non-linear magnetisation response of the MNPs. This condition is called non-linear ACS, where the harmonics generated due to the non-linear magnetisation of the MNPs are employed, and easily differentiated from the excitation frequency. There are two types of non-linear ACS; harmonic detection approach and the mixed-frequency detection approach [8-10]. In harmonic detection approach, a sufficient is used to fulfil the condition that the Langevin parameter is above two $(\xi>2)$, in which even the third harmonic can be identified [11]. Yoshida et al., (2012) developed a system for the detection of magnetic nanoparticles using the second-harmonic signal from the MNPs, using a simple inductive pickup coil gradiometer [12]. The advantage of using the second-harmonic signal is that the interference of the excitation field is significantly reduced. Furthermore, [13] developed an MPI system that uses harmonic signals of the MNPs by their non-linear magnetisation curve. Harmonic signals from the MNPs were measured when $\mu_{0} H_{a c}$ of 1 $m \mathrm{~T}$ is applied to the sample while $\mu_{0} H d c$ was varied from 0 to $5 m \mathrm{~T}$, and a solid-phase MNPs ware used.

Mixed-frequency detection approach, on the other hand, uses two different excitation fields $H_{01}$ and $H_{02}$, with $H_{01}$ having low frequency but with high amplitude and $H_{02}$ having high frequency but with small amplitude of excitation. Therefore, a response containing two frequencies is produced by the MNPs. This response is used in identifying the MNPs [14]. In MRX, the MNPs are exposed to a high magnetic excitation after an initial time $t . H$ is switched off and the decay in the magnetic signal generated by the MNPs (relaxation signal) is measured for $t>0$ using another magnetic field (pulse magnetic field) [15]. Magnetic relaxometry was used to detect breast cancer cells, using targeted MNPs and ultra-sensitive magnetic field sensors, in particular using superconducting quantum interference device (SQUID) sensors in [16]. The magnetic remanence-based approach is comparable with MRX, as MNPs with long Neel relaxation period are employed. Here, the MNPs response is retained for an elongated period to differentiate the positions at which the excitation and the signal measurement are made [17]. In this study, the harmonic detection approach is implemented in order to differentiate the harmonics easily by applying a sufficient excitation field amplitude.

\section{RESEARCH METHODS}

For the second harmonic signal detection to be conducted, an MPI scanner is required. For that reason, a single-sided MPI scanner developed by [18] is used in this study. The scanner is a one-dimensional scanner having $22.8 \mathrm{kHz}$ excitation frequency. In addition, Resovist (Fuji pharma) and Perimag (Micromod) MNPs were employed as the tracer materials, both immobilised and in liquid form to imitate their accumulation in the tumour region in real life SLNB. Explaining research chronological, including 
research design, research procedure (in the form of algorithms, Pseudocode or other), how to test and data acquisition [1-3]. The description of the course of research should be supported references, so the explanation can be accepted scientifically $[2,4]$.

\subsection{Single-Sided MPI Scanner Set-Up}

The measurement system for the second harmonic detection of MNPs is depicted in Figure 1, which consists of the constructed coils, arranged coaxially on the same plane to form the single-sided MPI scanner. The designed BPF and BSF, as well as the resonant circuit (for second harmonic detection) were also connected appropriately to the transmitting and receiving sections of the scanner device [19, 20, 21, 22]. Other equipment utilised in the scanner realisation is also shown in Figure 1. These equipment are the function generator (BK Precision 4055), AC power amplifier (HP 8447F H64), DC power supply (Tektronix PS280) and the DAQ device (NI USB-6009). Additionally, sheets of acrylic material (9 mm thickness) were also used to hold the coils coaxial arrangement in a fixed position. The acrylic material is selected due to its little weight and does not exert any magnetic field on the scanner operation. Furthermore, it has a reasonable degree of compatibility with human tissues.

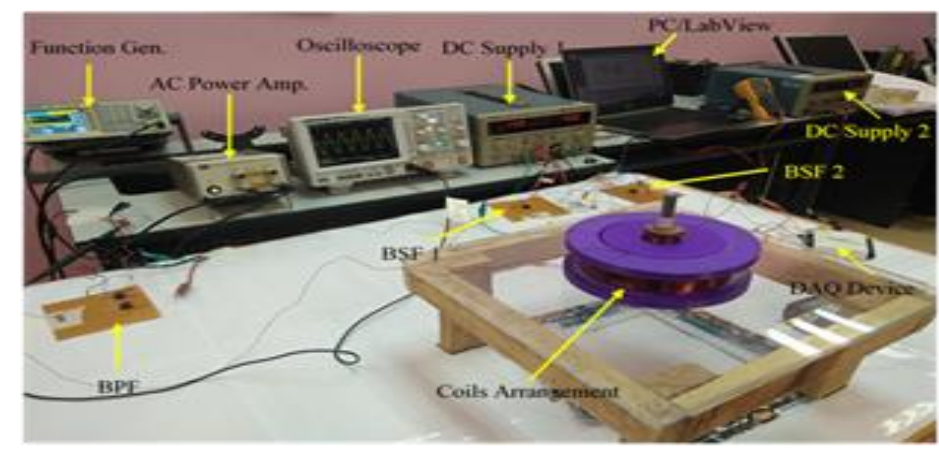

Figure 1. Measurement system set-up

The excitation field is generated by an alternating current of $1 \mathrm{~A}$, with an excitation frequency of $22.8 \mathrm{kHz}$, having an amplitude of $30 \mathrm{mV}$ peak-to-peak. Conversely, the selection field is generated with a direct current of $2 \mathrm{~A}$. The currents for the excitation and selection field were applied in the opposite direction, to achieve spatial encoding and the creation of the field free point. The parameters of the coils that made up the single-sided MPI scanner are summarised in Table 1.

Table 1. Coils Specifications

\begin{tabular}{lccc}
\hline \multicolumn{1}{c}{ Type } & $\begin{array}{c}\text { Excitation } \\
\text { Coil }\end{array}$ & $\begin{array}{c}\text { Selection } \\
\text { Field Coil }\end{array}$ & $\begin{array}{c}\text { Pickup } \\
\text { Coil }\end{array}$ \\
\hline $\begin{array}{l}\text { Number of turns } \\
\text { Number of layers }\end{array}$ & 352 & 416 & $208+208$ \\
$\begin{array}{l}\text { Wire diameter } \\
(\mathrm{mm})\end{array}$ & 8 & 9 & 8 \\
\hline
\end{tabular}

\subsection{Single-Sided MPI Scanner Set-Up}

As explained earlier, two commercially available MNPs were employed as the tracer materials, to be detected under the influence of the magnetic fields generated by the developed MPI scanner. Therefore, samples of Resovist (Fuji pharma) and Perimag (Micromod) particles were prepared for the second harmonic detection and inserted into one well of a 96-well cell culture plate (655-180, flat-bottom), with a total sample volume of $300 \mu \mathrm{l}$ assumed. The iron concentration in the MNPs found at the sentinel lymph node is estimated to be around $100 \mu \mathrm{g}$ [2]. Thus, $100 \mu \mathrm{g}$ Fe is employed as the iron concentration in the MNPs samples to be detected using second harmonic detection.

Initially, the samples were diluted with distilled water to achieve the desired volume in liquid form and mixed with glycerol to get the immobilized phase. Consequently, the volume of the MNPs needed to contain $100 \mu \mathrm{g}$ Fe is calculated using the particles parameters given in Table 2, as $3.6 \mu$ l for Resovist and 4.0 $\mu \mathrm{l}$ for Perimag. Finally, a volume of $300 \mu \mathrm{l}$ sample is attained by adding $296.4 \mu \mathrm{l}$ of distilled water to Resovist and $296 \mu \mathrm{l}$ of distilled water to Perimag MNPs. 


\begin{tabular}{cccc}
\multicolumn{2}{c}{ Table 2. Resovist and Perimag MNPs Parameters } \\
\hline MNPs & $\begin{array}{c}\text { Concentration } \\
(\mathrm{mg} \mathrm{Fe} / \mathrm{ml})\end{array}$ & $\begin{array}{c}\text { Density } \\
(\mathrm{g} / \mathrm{ml})\end{array}$ & $\begin{array}{c}\text { Calculated volume } \\
(\mu \mathrm{l})\end{array}$ \\
\hline $\begin{array}{c}\text { Resovist } \\
(60 \mathrm{~nm})\end{array}$ & 28 & 1.057 & 3.6 \\
$\begin{array}{c}\text { Perimag } \\
(130 \mathrm{~nm})\end{array}$ & 25 & 1.400 & 4.0 \\
\hline
\end{tabular}

The MNPs need to be experimented not only in fluid form but also in a solid phase (immobilised). The samples are required to be immobilised to imitate the interaction between the tissues and the MNPs as in real life scenario. In this regard, the MNPs suspensions were diluted with glycerol to prepare the immobilised samples. The glycerol serves as a coagulant, which changes the fluid into semi-solid form. Hence, Resovist and Perimag MNPs were mixed with $235 \mu \mathrm{l}$ and $235.2 \mu \mathrm{l}$ of glycerol respectively, to form an immobilised sample as shown in Figure 2.
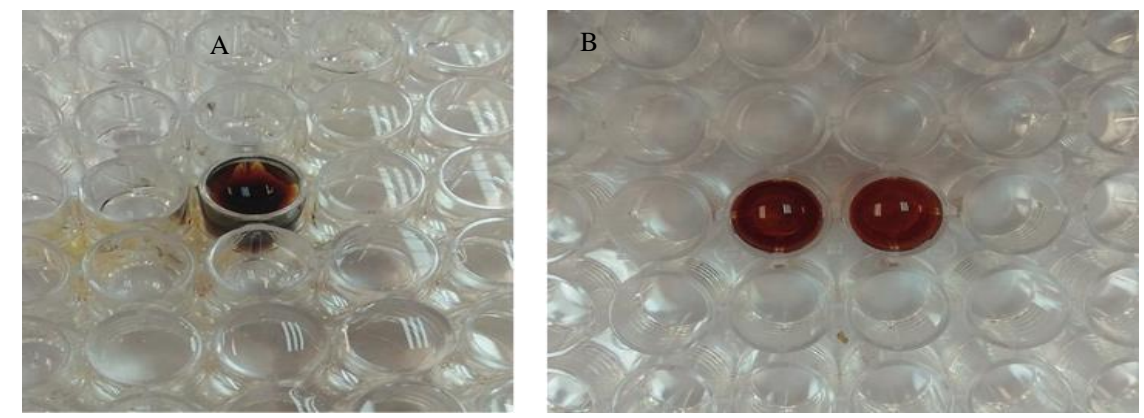

Figure 2. Immobilised MNPs samples (a) one sample perimag and (b) two sample resovist particles

\subsection{Experiment Performed}

The experiments to detect the tracer samples in liquid and solid form using second harmonic detection were conducted. For proper investigation on the harmonious features of the MNPs samples, the excitation magnetic field was fixed at $0.8,1.7,2.6,3.0,3.4$ and $4.4 \mathrm{mT}$ at $22.8 \mathrm{kHz}$ excitation frequency. Besides, the selection magnetic field was varied from 0 to $5 \mathrm{mT}$. Initially, the samples were placed under the scanner (one at a time), exposing them to the excitation magnetic field at the FFP by moving the sample to all the spatial positions across the FOV. Since the developed MPI scanner is one-dimensional, the FOV has a dimension of $60 \times 60 \mathrm{~mm}^{2}$ with a total of 169 spatial locations. Due to the influence of the excitation field, the MNPs samples became saturated and responded by producing another magnetic field. The particle's magnetisation response for each spatial position is recorded in the form of the voltage signal, at the second harmonic frequency. Measurements of the particles magnetisation were executed in the z-direction, where the excitation magnetic field is assumed to be highly uniform in strength and path. The right-hand rule for magnetic field produced by a solenoid states that the thumb points in the direction of the field, while the coiled fingers indicate the direction of current [23]. The magnetic field diminishes with increasing distance away from the coil. Therefore, the distance along the z-direction is varied to determine how far the second harmonic signals can be detected, which is related to the distance between the MPI scanner and the sentinel lymph node under examination. In this regard, the mean depth of the sentinel lymph node from the skin surface in humans is estimated to be $12 \pm 5 \mathrm{~mm}[2,24]$. Hence, the distance in the z-direction is varied from the surface of the pickup coil to about $30 \mathrm{~mm}$, so that the sentinel lymph node distance is appropriately covered. Lastly, the voltage induced in the pickup coil at each interval in the z-direction is recorded, with the second harmonic signal amplitude determined through FFT of the induced voltage signal, which is employed in the MNPs image reconstruction.

\section{RESULTS AND ANALYSIS}

Initially, the MNPs samples were placed (one after the other) at the centre of the designed singlesided MPI scanner for excitation and the corresponding MPI signal measurement. The vertical distance (z-direction) between the sample and the scanner is varied from the surface of the pickup coil to over $30 \mathrm{~mm}$ in space. The variation in the z-direction determines the sensitivity of the developed scanner with 
increasing distance. Liquid samples of Resovist and Perimag particles were prepared, each containing $100 \mu \mathrm{g}$ of the tracer material in a $300 \mu \mathrm{l}$ volume. Immobilised samples, on the other hand, contains $235 \mu 1$ of glycerol and the same tracer content as the liquid sample $(100 \mu \mathrm{g})$. The excitation magnetic field was generated with a periodic signal of $22.8 \mathrm{kHz}$, from an AC of $1 \mathrm{~A}$ while the DC for the selection field was set at $2 \mathrm{~A}$. As a result, the induced voltage at the second harmonic when the distance in the $\mathrm{z}$-direction is varied is depicted in Figure 3.

Perimag particles induced higher voltage signals as compared to Resovist particles up to a distance of $25 \mathrm{~mm}$, as shown in Figure 3. Furthermore, liquid samples produce greater voltage signals than the immobilised samples, which is due to more rapid decay of Neel relaxation times and the size difference of the MNPs as explained earlier. Thus, both Resovist and Perimag particles could be evidently detected at 25 $\mathrm{mm}$ in the $\mathrm{z}$-direction, which is adequate for tracer detection in SLNB application.

\subsection{Second Harmonic Signal Measurements}

Subsequently, the MNPs tracer sample is scanned through the FOV and the amplitude of the second harmonic induced signal is recorded, to determine the position of the MNPs sample in the XY-plane by plotting its contour map. Before the contour plot, the dependence of the second harmonic signal with varying the DC magnetic field for the selection field $\left(\mathrm{H}_{\mathrm{dc}}\right)$ is investigated. The $\mathrm{H}_{\mathrm{dc}}$ is varied between $\pm 25 \mathrm{mT}$ to have signals induced from both the positive and negative magnetisation of the MNPs, while the AC excitation field is fixed at $10 \mathrm{mV}$ peak-to-peak from $22.8 \mathrm{kHz}$. Figure 4 depicts the second harmonic signals recorded from varying Hdc for Resovist sample. It can be deduced that the signal amplitude for liquid Resovist sample reaches its peak of $15 \mathrm{mV}$ at around $5 \mathrm{mT} \mathrm{Hdc}$, while the immobilised sample has a peak signal amplitude of approximately $13 \mathrm{mV}$ at the same DC magnetic field.

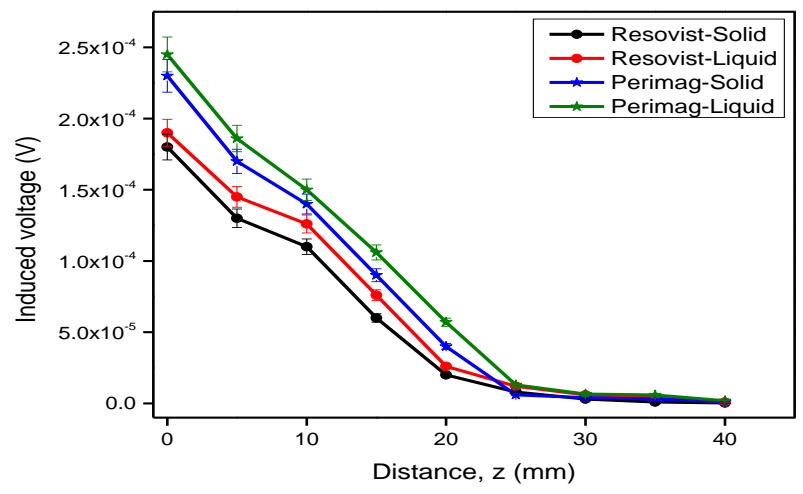

Figure 3. Induced voltage with varying distance along z-direction

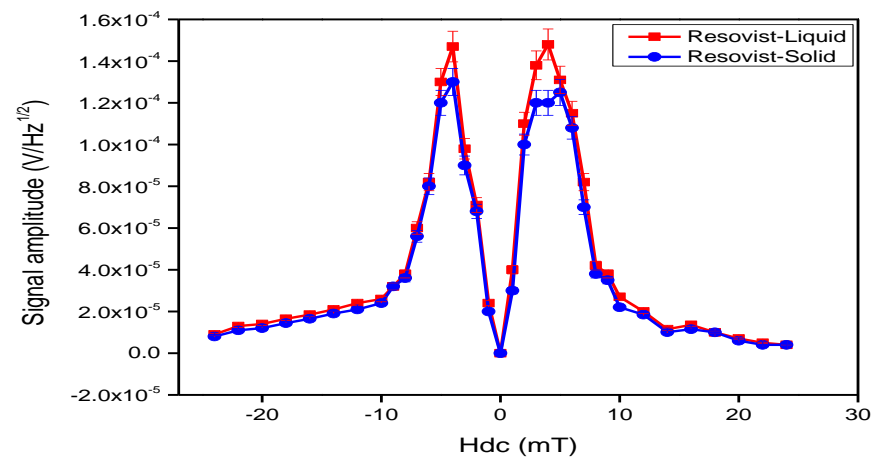

Figure 4. Second harmonic signal dependence on different DC magnetic field for Resovist sample

The second harmonic signal dependence with varying DC magnetic field for Perimag sample was also investigated and is presented in Figure 5. Liquid Perimag sample generates peak signal amplitude of around $15.8 \mathrm{mV}$ as compared to $14 \mathrm{mV}$ produced by the immobilised Perimag particles at $\pm 6 \mathrm{mT}$ DC magnetic field. For symmetry and compliance with the AC excitation magnetic field applied in both negative 
and positive sides of the particles magnetisation curve, the DC magnetic field is also applied in a negative and positive direction, to have signals induced in both directions.

It is therefore evident from the measurements conducted on the four samples that the dynamic region of Resovist and Perimag particle magnetisation curve is within $\pm 5 \mathrm{mT}$ DC magnetic field for Resovist and $6 \mathrm{mT}$ DC magnetic field for Perimag, after which no further magnetisation is recorded, as revealed by Figure 4 and Figure 5. This implies that the magnetisation response of the tracers is confined to the FFP by the selection field at $\pm 5 \mathrm{mT}$ for Resovist and $\pm 6 \mathrm{mT}$ for Perimag particles. Additionally, the voltage signal induced by the tracers is purely contributed by the tracers within these DC magnetic fields.

Next, the dependence of the AC excitation magnetic field on the induced signal is explored, by varying the excitation field between $0-10 \mathrm{mT}$ while the DC selection field was kept constant at $5 \mathrm{mT}$. The results obtained revealed that the signal amplitude of the second harmonic frequency increases exponentially with increasing Hac, as shown in Figure 6. For Resovist particles, similar signal amplitudes were recorded for $\mathrm{H}_{\mathrm{ac}} \leq 2 \mathrm{mT}$, as a result of similar averaged relaxation time experienced by the liquid and immobilised samples, in addition to being under the same temperature condition.

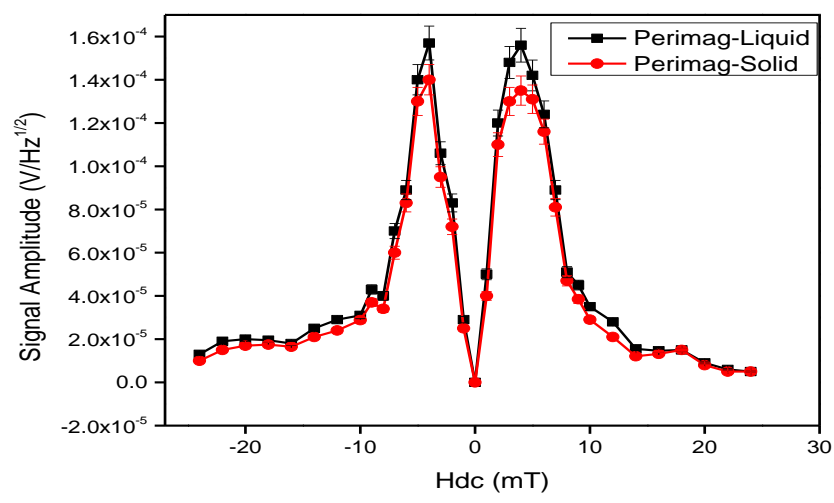

Figure 5. Second harmonic signal dependence on different DC magnetic field for Perimag sample

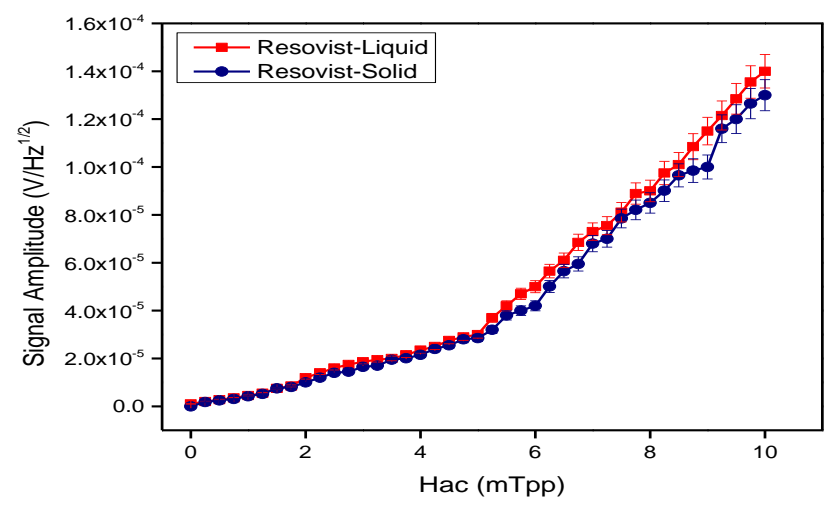

Figure 6. Dependence of excitation magnetic field on the induced signal for resovist

Perimag particles, on the other hand, records different signal amplitude even at low excitation field, with the liquid sample generating higher harmonics than the immobilised sample as depicted in Figure 7. The various signal amplitudes obtained in the measurement is as a result of faster Neel relaxation time of the liquid sample as compared to that of the immobilised sample, due to the mean time between two flips of the particle's internal magnetisation. The signal amplitudes recorded for Perimag samples show a similar trend to that obtained from Resovist samples, with the exponential rise [25, 26].

The dependence of DC magnetic field on the excitation magnetic field for the peak second harmonic signal detection is finally investigated. As can be seen in Figure 4 and Figure 5, zero second harmonic signal amplitude was recorded when $\mathrm{H}_{\mathrm{dc}}$ is zero for both Resovist and Perimag particles but reaches its peak value when $\mathrm{H}_{\mathrm{dc}}$ increases. Figure 8 revealed a virtually linear relationship between $\mathrm{H}_{\mathrm{dc}}$ and $\mathrm{H}_{\mathrm{ac}}$, as 
the small value of $\mathrm{H}_{\mathrm{dc}}$ peak could be obtained with little $\mathrm{H}_{\mathrm{ac}}$ when the highest amount of the signal amplitude is recorded $[12,13,26-28]$.

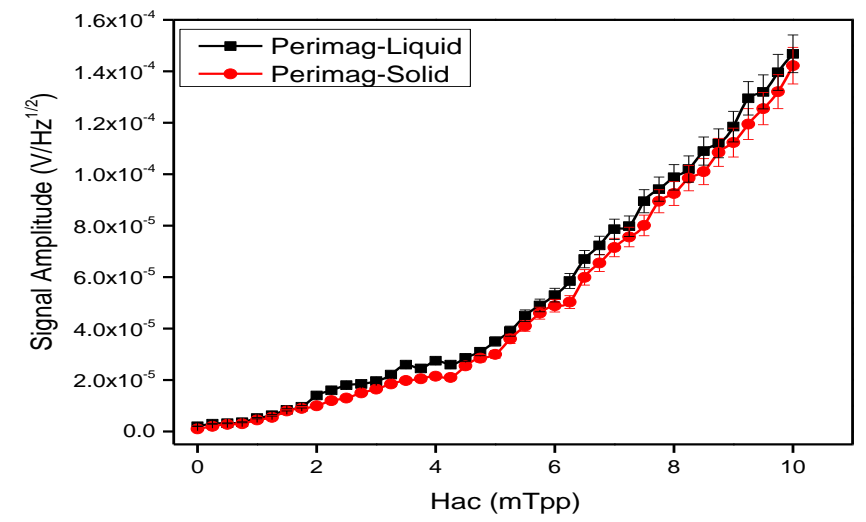

Figure 7. Dependence of second harmonic signal amplitude on AC excitation magnetic field for Perimag

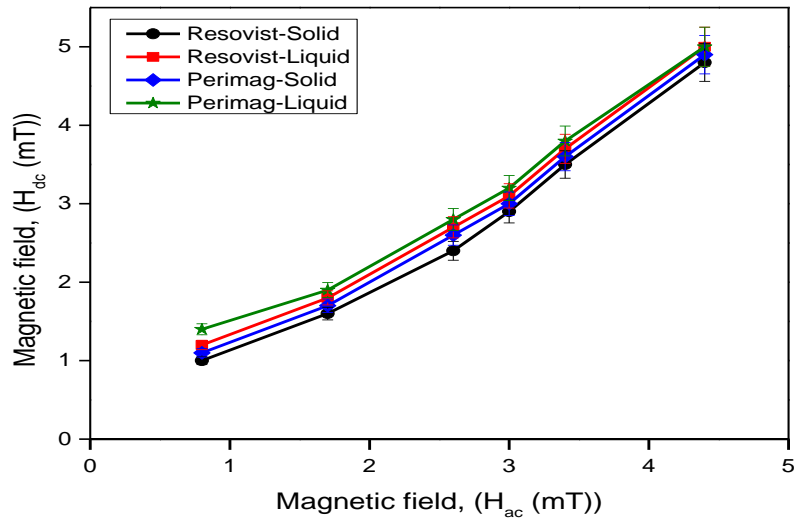

Figure 8. Relationship between $\mathrm{H}_{\mathrm{dc}}$ and $\mathrm{H}_{\mathrm{ac}}$ at peak signal amplitude

\section{CONCLUSION}

It has been shown that MPI signal with high SNR could be appropriately acquired from the nonlinear magnetization response of the tracers, using low frequency excitation magnetic field. The use of Bandstop filter removes the component of the excitation field, yielding a high SNR signal completely from tracer materials. Additionally, the simple resonant circuit designed for the second harmonic signal detection was found to be appropriate in extracting the MPI signal at the second harmonic frequency. Measurement results obtained clearly revealed the width of the dynamic range of Resovist and Perimag nanoparticles, with the latter producing higher signal amplitudes in all experiments, due to its large hydrodynamic diameter. Liquid samples were found to produce higher signal amplitudes than the immobilised samples due the dominance of Brownian rotation over Neel rotation of the particles. Thus, the outcome of this research showed that the MPI signal acquisition approach has a potential in the detection of tracers, which could help in obtaining high resolution tomographic images, in sentinel lymph node biopsy (SLNB) for breast cancer diagnosis.

\section{ACKNOWLEDGMENT}

This research was conducted with financial support by ORICC, Universiti Tun Hussein Onn Malaysia through Tier-1 Research Grant Vot Number H182 and Postgraduate Research Grant (GPPS) Vot Number U589. 


\section{REFERENCES}

[1] Gleich, and J. Weizenecker, "Tomographic imaging using the nonlinear response of magnetic particles," Nature, vol. 435, pp. 1214-1217, 2005.

[2] N. B. Othman, "Magnetic Nanoparticle Imaging for Biomedical Applications," unpublished.

[3] A. A. Sadiq, N. B. Othman, and M. M. Abdul Jamil, "Magnetic particle imaging system for cancer diagnosis: an overview," ARPN Journal of Engineering and Applied Sciences, vol. 10, pp. 8556-8561, 2015.

[4] T. M. Buzug, G. Bringout, M. Erbe, K. Gräfe, M. Graeser, M. Grüttner, and J. Haegele, "Magnetic particle imaging: an introduction to imaging and hardware realisation," Zeitschrift für Medizinische Physik, vol. 22, pp. 323-334, 2012.

[5] B. Gleich, Principles and applications of magnetic particle imaging. Springer Science \& Business Media, Germany, 2013.

[6] S. Tanaka, H. Murata, T. Oishi, Y. Hatsukade, Y. Zhang, H. Horng, and H. C. Yang, "Imaging of magnetic nanoparticles using the second harmonic of magnetization with a DC bias field," IEEE Transactions on Magnetics, vol. 51, pp. 1-4, 2015.

[7] K. Enpuku, Y. Tsujita, K. Nakamura, T. Sasayama, and T. Yoshida, "Biosensing utilizing magnetic markers and superconducting quantum interference devices,” Superconductor Science and Technology, vol. 30, pp. 1-12, 2017.

[8] T. Mizoguchi, A. Kandori, R. Kawabata, K. Ogata, T. Hato, A. Tsukamoto, and K. Enpuku, "Highly sensitive third-harmonic detection method of magnetic nanoparticles using an AC susceptibility measurement system for liquid-phase assay," IEEE Transactions on Applied Superconductivity, vol. 26, pp. 1-4, 2016.

[9] T. Morishige, T. Mihaya, S. Bai, T. Miyazaki, T. Yoshida, M. Matsuo, and K. Enpuku, "Highly sensitive Magnetic Nanoparticle Imaging using Cooled-Cu/HTS-Superconductor Pickup coils," IEEE Transaction on Applied Superconductivity, vol. 24, pp 105-109, 2014.

[10] T. Sasayama, Y. Tsujita, M. Morishita, M. Muta, T. Yoshida, and K. Enpuku, "Three-dimensional magnetic nanoparticle imaging using small field gradient and multiple pickup coils," Journal of Magnetism and Magnetic Materials, vol. 427, pp. 143-149, 2017.

[11] C. C. Yang, S. Y. Yang, H. H. Chen, W. L. Weng, H. E. Horng, J. J. Chieh, C. Y. Hong, and H. C. Yang, "Effect of molecule-particle binding on the reduction in the mixed frequency alternating current magnetic susceptibility of magnetic bio-reagents," Journal of Applied Physics, vol. 112, pp. 024704, 2012.

[12] T. Yoshida, N. B. Othman, T. Tsubaki, J. Takamiya, and K. Enpuku, "Evaluation of Harmonic Signals for the detection of magnetic Nanoparticles," IEEE Transaction on Magnetics, vol. 48, pp. 3788-3791, 2012.

[13] N. B. Othman, T. Tsubaki, T. Yoshida, K. Enpuku, and A. Kandori, "Magnetic Nanoparticle Imaging Using Harmonic Signals," IEEE Transaction on Magnetics, vol. 48, pp. 3776-3779, 2012.

[14] K. Enpuku, Y. Ueoka, T. Sakakibara, M. Ura, T. Yoshida, T. Mizoguchi, and A. Kandori, "Liquid-phase immunoassay utilizing binding reactions between magnetic markers and targets in the presence of a magnetic field," Applied Physics Express, vol. 7, pp. 097001, 2014.

[15] C. Johnson, N. L. Adolphi, K. L. Butler, D. M. Lavato, R. Larson, P. Schwindt, and P. R. Flynn, "Magnetic relaxometry with an atomic magnetometer and SQUID sensors on targeted cancer cells," Journal of Magnetism and Magnetic Materials. Vol. 24, pp. 2613-2619, 2012.

[16] H. J. Hathaway, K. S. Butler, N. L. Adolphi, D. M. Lovato, R. Belfon, D. Fegan, and D. L. Huber, "Detection of breast cancer cells using targeted magnetic nanoparticles and ultra-sensitive magnetic field sensors," Breast Cancer Research, vol. 13, pp. R108, 2011.

[17] S. Uchida, Y. Higuchi, Y. Ueoka, T. Yoshida, K. Enpuku, S. Adachi, K. Tanabe, A. Tsukamoto, and A. Kandori, "Highly sensitive liquid-phase detection of biological targets with magnetic markers and high Tc SQUID," IEEE Transactions on Applied Superconductivity, vol. 24, pp. 1600105, 2014.

[18] A. A. Sadiq, M. M. Abdul Jamil, and N. B. Othman, "Single-Sided Magnetic Particle Imaging using Perimag Magnetic Nanoparticles," Journal of Telecommunication, Electronic and Computer Engineering, submitted for publication.

[19] A. A. Sadiq, N. B. Othman, and M. M. Abdul Jamil, "Excitation coil design for single-sided magnetic particle imaging scanner, ” In IEEE Asia-Pacific Conference on Applied Electromagnetics, pp. 364-368, Dec. 2016.

[20] A. A. Sadiq, N. B. Othman, and M. M. Abdul Jamil, "Computational and Experimental Study of Gradiometer Coil Sensor Design for Single-Sided Magnetic Particle Imaging, ” In Journal of Physics: Conference Series, vol. 1049, No. 1, pp. 012068, Jul. 2018.

[21] A. A. Sadiq, N. B. Othman, M. M. Abdul Jamil, M. Youseffi, M. Denyer, W. W. Zakaria, and M. M. Tomari, "Fourth-Order Butterworth Active Bandpass Filter Design for Single-Sided Magnetic Particle Imaging Scanner." Journal of Telecommunication, Electronic and Computer Engineering, vol. 10, no. 1-17, pp. 17-21, 2018.

[22] F. T. Sattel, T. Knopp, S. Biederer, B. Gleich, J. Weizenecker, J. Borgert, and T. Buzug, "Single-sided device for magnetic particle imaging," Journal of applied physics, vol. 42, no. 2, pp. 1-5, 2009.

[23] A. Pathak, "An elementary argument for the magnetic field outside a solenoid," European Journal of Physics, vol. 38, no. 1, pp. 015201, 2016.

[24] K. H. Song, C. Kim, C. M. Cobley, Y. Xia, and L. V. Wang, "Near-infrared gold nanocages as a new class of tracers for photoacoustic sentinel lymph node mapping on a rat model," Nano letters, vol. 9, no. 1, pp. 183-188, 2008.

[25] Y. Zhang, H. Murata, Y. Hatsukade, and S. Tanaka, "Superparamagnetic nanoparticle detection using second harmonic of magnetisation response" Review of scientific instruments, vol. 84, no. 9, pp. 094702, 2013. 
[26] S. Waanders, M. Visscher, R. R. Wildeboer, T. O. B. Oderkerk, H. J. Krooshoop, and B. T. Haken, "A handheld SPIO-based sentinel lymph node mapping device using differential magnetometry," Physics in medicine and biology, vol. 61, no. 22, pp. 8120-8134, 2016.

[27] K. Gräfe, M. Weber, T. Sattel, and T. Buzug, "The precision of an MPI Scanner Construction: Registration of Measured and Simulated Magnetic Fields," Biomedical Engineering/Biomedizinische Technik, vol. 58, no. 1, pp. 1$2,2013$.

[28] T. Knopp, and T. M. Buzug, Magnetic particle imaging: an introduction to imaging principles and scanner instrumentation. Springer Science \& Business Media, Berlin, 2012.

\section{BIOGRAPHIES OF AUTHORS}
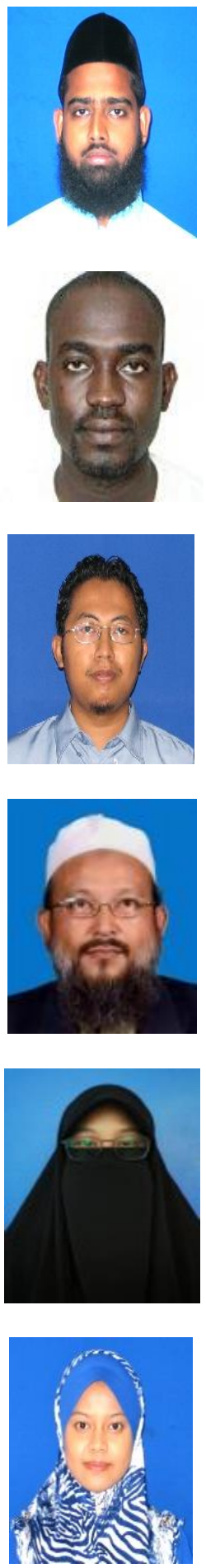

Ts. Dr. Muhammad Mahadi Bin Abdul Jamil is an Associate Professor at Universiti Tun Hussein Onn Malaysia. He is holding a PhD of Electronic Engineering in Biomedical Engineering from University of Bradford, UK.

Abdulkadir Abubakar Sadiq is a student who is currently undertaking his PhD study program under Faculty of Electric and Electronic Engineering, UTHM, Johor.

Ts. Muhammad Shukri Ahmad is a Senior Lecturer at Department of Electrical and Electronic Engineering, Universiti Tun Hussein Onn Malaysia. He is holding a Master of Electrical Engineering from Technical College Universiti Tun Hussein Onn, Malaysia.

Noordin Asimi Mohd Noor is an Associate Professor at Faculty of Innovative Design and Technology, Universiti Sultan Zainal Abidin.

Nur Adilah Abd Rahman is a student who is currently undertaking her PhD study program under Faculty of Electric and Electronic Engineering, UTHM, Johor. She is holding a Master of Electrical Engineering in Biomedical Engineering from Universiti Tun Hussein Onn Malaysia.

Dr. Nurmiza Othman is a Senior Lecturer at Universiti Tun Hussein Onn Malaysia. She is holding a PhD of Engineering in Electrical and Electronic Engineering from Kyushu University, Japan. 